\title{
Western Blot for Detecting Phosphorylated STAT3
}

Huagang Zhang*

Albert Einstein College of Medicine, Yeshiva University, New York City, USA

*For correspondence: huagangzhang@gmail.com

[Abstract] The STAT3 transcription factor is an important signaling molecule for many cytokines and growth factor receptors and is constitutively activated in a number of human tumors and possesses oncogenic potential and anti-apoptotic activities. STAT3 is activated by phosphorylation at Tyr705, which induces dimerization, nuclear translocation, and DNA binding. Western blot is most commonly used to detect the activation of STAT3 by using an antibody that is specific for the phosphorylated tyrosine 705 .

\section{Materials and Reagents}

1. Tumor cell lines with constitutive activation of STAT3 (positive control).
a. DU145 (ATCC, catalog number: HTB-81 ${ }^{\text {TM }}$ )
b. HepG2 (ATCC, catalog number: HB-8065 ${ }^{\mathrm{TM}}$ )
c. Hep3B (ATCC, catalog number: HB-8064 ${ }^{\text {TM }}$ )
d. Huh7

2. Phospho-Stat3 (Tyr705) (D3A7) ХРтм Rabbit (Cell Signaling Technology, catalog number: 9145)

3. Stat3 (124H6) Mouse mAb (Cell Signaling Technology, catalog number: 9139)

4. HRP Goat Anti-Rabbit I (BD Biosciences, catalog number: 554021)

5. $\quad \beta$-Actin (13E5) Rabbit mAb (Cell Signaling Technology, catalog number: 4970)

6. Anti-mouse IgG, HRP-linked Antibody (Cell Signaling Technology, catalog number: 7076) Note: The above antibodies have been tested by the author and may be substituted with the antibodies desired by users.

7. Phosphate buffered saline (PBS)

8. 1x halt protease and phosphatase inhibitor cocktail (Thermo Fisher Scientific, catalog number: 78440)

9. M-PER mammalian protein extraction reagent (Thermo Fisher Scientific, catalog number: 78501)

10. Bio-Rad protein assay dye reagent concentrate (Bio-Rad Laboratories, catalog number: 500-0006)

11. 10x Tris/Glycine/SDS (Bio-Rad Laboratories, catalog number: 161-0771) 
12. Methanol (Thermo Fisher Scientific, catalog number: A412-20)

13. Tris buffered saline (Bio-Rad Laboratories, catalog number: 170-6435)

14. Tween-20 (Santa Cruz Biotechnology, catalog number: sc-29113)

15. Bovine serum albumin (BSA) (MP Biomedicals, catalog number: 810033)

16. Supersignal west Dura extended duration substrate (Thermo Fisher Scientific, catalog number: 34075)

17. Precision plus protein dual color standards (Bio-Rad Laboratories, catalog number: 1610374)

18. Restore plus western blot stripping buffer (Thermo Fisher Scientific, catalog number: 46430)

19. Protein lysis buffer (see Recipes)

20. Electrophoresis buffer (see Recipes)

21. 1x Tris buffered saline (TBS) (see Recipes)

22. Transfer buffer (see Recipes)

23. Blocking buffer (see Recipes)

24. Wash buffer (see Recipes)

25. Primary antibody dilution buffer (see Recipes)

26. Blotting membrane (see Recipes)

\section{Equipment}

1. Microcentrifuges (Eppendorf, model: $5415 \mathrm{R}$ )

2. Thermolyne Rotomix (BioSurplus, model: 50800)

3. Microcentrifuge tubes

4. Nitrocellulose or PVDF membrane

5. SmartSpec plus spectrophotometer (Bio-Rad Laboratories)

\section{Procedure}

A. Protein blotting

1. Treat cells by adding fresh media containing regulator for desired time.

2. Aspirate media from cultures; wash cells with 1x PBS; aspirate.

3. Lyse cells by adding $1 x$ protein lysis buffer.

4. Immediately scrape the cells off the plate and transfer the extract to a microcentrifuge tube.

5. Incubate at room temperature (RT) for $5 \mathrm{~min}$.

6. Microcentrifuge for $10 \mathrm{~min}$ at $13,000 \mathrm{rpm}$. 
7. Transfer supernatant to a clean microcentrifuge tube.

8. Measure the protein concentration using the Bio-Rad protein assay dye reagent.

9. Heat a $20 \mu \mathrm{g}$ sample to $95-100^{\circ} \mathrm{C}$ for $10 \mathrm{~min}$; cool on ice.

10. Load $20 \mu \mathrm{g}$ sample onto SDS-PAGE gel $(10 \mathrm{~cm} \times 10 \mathrm{~cm})$ and load $7 \mu \mathrm{l}$ precision plus protein fual color standards to determine molecular weights.

11. Electrophoresis at constant 80 Volts until the protein dye reaches the bottom of the gel.

12. Electrotransfer to nitrocellulose or PVDF membrane using transfer buffer (constant 30 Volts overnight at $4{ }^{\circ} \mathrm{C}$ ).

B. Membrane blocking and detection of pSTAT3

1. Incubate membrane in $25 \mathrm{ml}$ of blocking buffer for $1 \mathrm{~h}$ at $\mathrm{RT}$.

2. Wash three times for 5 min each with $15 \mathrm{ml}$ of TBS/T.

3. Incubate membrane and primary phospho-Stat3 (Tyr705) (D3A7) XP'M rabbit antibody $(1: 1,000)$ in $10 \mathrm{ml}$ primary antibody dilution buffer with gentle agitation overnight at $4{ }^{\circ} \mathrm{C}$.

4. Wash three times for 5 min each with $15 \mathrm{ml}$ of TBS/T.

5. Incubate membrane with HRP-conjugated goat anti-rabbit secondary antibody $(1: 5,000)$ and HRP-conjugated anti-biotin antibody $(1: 1,000)$ to detect biotinylated protein markers in $10 \mathrm{ml}$ of blocking buffer with gentle agitation for $1 \mathrm{~h}$ at RT.

6. Wash three times for 5 min each with $15 \mathrm{ml}$ of TBS/T.

7. Incubate membrane with $4 \mathrm{ml}$ Supersignal west dura extended duration substrate with gentle agitation for 5 min at RT.

8. Drain membrane of excess developing solution (do not let dry), wrap in plastic wrap and expose to x-ray film. An initial $10 \mathrm{sec}$ exposure should indicate the proper exposure time.

C. Detection of STAT3

1. Wash the membrane three times for $5 \mathrm{~min}$ each with $15 \mathrm{ml}$ of TBS/T.

2. Incubate membrane with $10 \mathrm{ml}$ restore plus western blot stripping buffer for $15 \mathrm{~min}$ to stripe the bonding antibodies.

3. Wash the membrane three times for 5 min each with $15 \mathrm{ml}$ of TBS/T.

4. Repeat steps 11-18 and use Stat3 (124H6) mouse mAb as primary antibody and HRPconjugated anti-mouse IgG as secondary antibody.

D. Detection of $\beta$-actin

1. Repeat steps $19-22$ for $\beta$-actin detection.

E. Analyzing the level of STAT3 activation 
1. Scan the film and measure the intensity of pSTAT3 and STAT3 bands. Calculate the STAT3 activation using the following formula:

Percentage of STAT3 activation $=($ intensity of pSTAT3) $/$ (intensity of STAT3 + intensity of pSTAT3) $\times 100 \%$.

\section{$\underline{\text { Recipes }}$}

1. Electrophoresis buffer

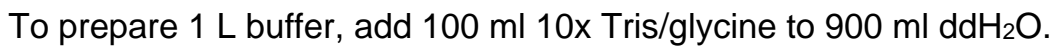

2. Protein lysis buffer

M-PER mammalian protein extraction reagent with $1 \mathrm{x}$ halt protease and phosphatase inhibitor cocktail.

3. Transfer buffer

To prepare $1 \mathrm{~L}$ buffer, add $100 \mathrm{ml}$ 10x Tris/Glycine/SDS and $200 \mathrm{ml}$ methanol to $700 \mathrm{ml}$ $\mathrm{ddH}_{2} \mathrm{O}$.

4. $1 \times$ TBS

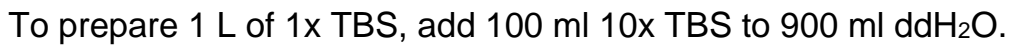

5. Blocking buffer

1x TBS, 0.1\% tween-20 with 5\% (w/v) BSA.

6. Wash buffer (TBS/T)

$1 x$ TBS, $0.1 \%$ tween-20

7. Primary antibody dilution buffer

1x TBS, $0.1 \%$ tween-20 with $5 \%$ BSA

8. Blotting membrane

This protocol has been optimized for nitrocellulose membranes, which CST recommends. PVDF membranes may also be used.

\section{References}

1. Campbell, C. L., Jiang, Z., Savarese, D. M. and Savarese, T. M. (2001). Increased expression of the interleukin-11 receptor and evidence of STAT3 activation in prostate carcinoma. Am J Pathol 158(1): 25-32.

2. Fujimoto, M., Naka, T., Nakagawa, R., Kawazoe, Y., Morita, Y., Tateishi, A., Okumura, K., Narazaki, M. and Kishimoto, T. (2000). Defective thymocyte development and perturbed homeostasis of T cells in STAT-induced STAT inhibitor-1/suppressors of cytokine signaling-1 transgenic mice. J Immunol 165(4): 1799-1806. 